\title{
DETERMINAÇÃO DO POSICIONAMENTO ÓTIMO DE APOIOS EM PONTES
}

\author{
OPTIMUM SUPPORT POSITIONS IN BRIDGES
}

\author{
Moacir Kripka ${ }^{1}$, Luttgardes de Oliveira Neto ${ }^{2}$, Rosana Maria Luvezute Kripka ${ }^{3}$ \\ ${ }^{1}$ Programa de Pós-graduação em Engenharia Civil e Ambiental, Universidade de Passo Fundo, Passo Fundo, \\ RS, E-mail: mkripka@upf.br \\ ${ }^{2}$ Departamento de Engenharia Civil e Ambiental, UNESP, Bauru, SP, E-mail: lutt@feb.unesp.br \\ ${ }^{3}$ Instituto de Ciências Exatas e Geociências, Universidade de Passo Fundo, Passo Fundo, RS, E-mail: \\ rkripka@upf.br
}

\begin{abstract}
RESUMO
O presente trabalho apresenta um estudo para a determinação do posicionamento otimizado dos apoios em pontes, bem como da relação ótima entre os vãos de vigas de pontes visando conduzir tanto a redução como a uniformização dos esforços nestas estruturas. O problema matemático foi formulado tendo como objetivo a minimização dos momentos fletores máximos, tendo como variáveis de projeto as coordenadas relativas de cada apoio interno. Na formulação as coordenadas nodais são canalizadas, uma vez que podem existir limitações à livre locação dos apoios. O programa desenvolvido com base neste estudo determina os esforços em cada apoio, para o deslocamento da carga ao longo da estrutura, a partir do Método dos Deslocamentos para análise de estruturas reticuladas, efetuando a otimização com o emprego de técnica de programação matemática. As estruturas analisadas a partir da implementação computacional do modelo idealizado apontam para a validação e eficiência do procedimento proposto.

Palavras-chave: otimização, pontes, posicionamento dos apoios.
\end{abstract}

\section{ABSTRACT}

This paper presents a study to determine the optimal support positions in bridges, as well as the optimal relation between the spans of beams, aiming both the reduction and the unifirmization of the efforts in these structures. The mathematical problem is formulated in order to minimize the maximum bending moments, taking the coordinate of each internal support as design variable. To take into account possible location limitations, size constraints are also considered in the formulation. The software developed based on this study determines the efforts in each support for load shifting along the structure by the usage of the Displacement Method, and the optimization is performed with a mathematical programming technique. The structures analyzed from the computational implementation of the idealized model point to the validation and efficiency of the proposed procedure.

Keywords: optimization, bridges, support positions.

\section{1 - INTRODUÇÃO}

Tanto a análise como o dimensionamento de estruturas, de modo geral, se constituem em procedimentos de grande complexidade. Ao envolverem um número significativo de variáveis, necessitam ser efetuados de maneira iterativa, ou seja, por meio de aproximações sucessivas. Em função deste caráter iterativo, os valores inicialmente arbitrados para as variáveis dependem de forma determinante tanto da sensibilidade como da experiência prévia do calculista. Ainda assim, o número de repetições exigido seria demasiadamente elevado caso se desejasse obter os valores ideais (ou "ótimos"), dentro do intervalo de resultados aceitáveis. Descrevendo, porém, o comportamento físico da estrutura por meio de funções matemáticas, buscam-se valores extremos de tais funções com o emprego de técnicas de otimização.

Nas últimas décadas, devido às crescentes facilidades computacionais, diversos estudos vêm sendo desenvolvidos no sentido de se abandonar os processos simplificados de cálculo, buscando gradativamente a adoção de modelos que reproduzam de forma mais realista o comportamento das estruturas. Os resultados destes estudos têm sido gradativamente incorporados aos softwares comerciais e, em consequência, ao dia-a-dia dos escritórios de projetos. No entanto, pouca ou nenhuma ênfase tem sido dada à sistematização do processo de idealização da estrutura, mesmo que em estudos acadêmicos. Como consequência, esta etapa continua sendo efetuada essencialmente de forma empírica, sendo que, uma vez idealizada uma configuração inicial, raramente são estudadas outras configurações possíveis, dentre um universo de alternativas também viáveis e eventualmente mais econômicas.

Não é de conhecimento dos autores a existências de estudos relacionados ao posicionamento ótimo dos apoios em pontes, apesar dos resultados obtidos a partir dos primeiros trabalhos com a inclusão das coordenadas nodais como variáveis de projeto terem sido considerados bastante promissores. Dentre esses destacam-se os estudos de Kripka e Antunes (1998) e Kripka e Antunes (2001), aplicados ao posicionamento dos apoios em vigas e grelhas de edifícios, respectivamente; de Wang, Jiang e Zhang (2004), visando maximizar a frequência natural em vigas e 
placas, e de Wang (2006), aplicado a pórticos planos. Os poucos trabalhos nessa linha, de acordo com Imam e AlShiri (1996), se devem basicamente à complexidade dos problemas que envolve a otimização geométrica, além de outras razões de natureza prática, entre elas o fato de que a otimização estrutural é vista quase que universalmente apenas como uma ferramenta para obter o mínimo peso de uma estrutura. Sob este enfoque, a economia obtida em relação ao custo total do projeto pode não ser suficientemente encorajadora para incentivar o aprofundamento dos estudos. Esta opinião é compartilhada por diversos autores, entre os quais Spillers (1975) e Kirsch (1991). Como reforço à visão dos autores citados, destaca-se o trabalho de Cohn e Dinovitzer (1994) o qual, com base em mais de 500 exemplos retirados de artigos e livros publicados no período de maior evolução da otimização estrutural, enfatiza a grande preocupação com aspectos matemáticos envolvidos, e em consequência a grande contribuição dada ao desenvolvimento das técnicas. Em contrapartida, o número de exemplos é muito pequeno em relação ao número de artigos e livros, sendo estes exemplos normalmente de interesse puramente acadêmico, em detrimento da aplicabilidade prática.

Este trabalho tem como proposta a otimização de esforços em superestruturas de pontes, analisadas como vigas independentes. Considerando a existência de uma certa liberdade de posicionamento dos apoios, já consideradas as limitações físicas impostas pelos obstáculos busca-se, com a mudança nas posições inicialmente idealizadas para os apoios, a redistribuição dos esforços pela alteração na rigidez relativa entre os vãos da viga, de modo a possibilitar maior uniformização destes esforços pela redução dos momentos máximos e, consequentemente, maior economia no consumo dos materiais estruturais.

\section{2 - CONCEITOS BÁSICOS}

O trabalho desenvolvido consiste na associação de técnica de otimização com a análise matricial de estruturas. Sendo assim, na sequência do presente item são apresentados alguns conceitos básicos necessários ao entendimento da metolodogia empregada no desenvolvimento do estudo.

\subsection{Otimização}

De forma genérica, um problema de otimização (no caso, minimização) pode ser expresso da seguinte forma:

$\begin{array}{ll}\text { Minimizar } & F\left(x_{i}\right) \quad \mathrm{i}=1 \ldots \mathrm{n} \\ \text { Sujeito a } & \mathrm{g}_{\mathrm{j}}\left(\mathrm{x}_{\mathrm{i}}\right) \leq 0 \quad \mathrm{j}=1 \ldots \mathrm{m} \\ & \mathrm{h}_{\mathrm{k}}\left(\mathrm{x}_{\mathrm{i}}\right)=0 \quad \mathrm{k}=1 \ldots 1 \\ & \mathrm{x}_{\mathrm{i}}{ }^{1} \leq \mathrm{x}_{\mathrm{i}} \leq \mathrm{x}_{\mathrm{i}}{ }^{\mathrm{u}}\end{array}$

Em que: $F$ designa a função objetivo e $X=\left(x_{1}, x_{2}, \ldots\right.$ $\left.x_{n}\right){ }^{T}$ consiste no vetor das variáveis de projeto. As demais funções são as chamadas restrições do problema (respectivamente, restrições de desigualdade, de igualdade e restrições laterais ou canalizadas, sendo estas últimas função dos limites inferior $l$ e superior $u$ que podem ser assumidos pelas variáveis). As funções envolvidas no problema podem conter as variáveis de projeto de forma explícita ou não, além de poderem ser desenvolvidas numérica ou analiticamente. Tanto a função objetivo como as funções de restrição podem ser lineares ou não lineares.

A forma apresentada para a descrição de um problema de otimização não é única, sendo encontradas diversas outras formas na literatura. Exemplificando, a inequação (4) é normalmente representada por duas restrições de desigualdade como as indicadas em (2). Porém, da maneira como é aqui apresentada, ficam caracterizados com maior clareza os limites que podem ser assumidos pelas variáveis de projeto.

As técnicas de otimização necessitam a priori da especificação de valores iniciais para as variáveis de projeto. Uma vez especificados estes valores, os mesmos são atualizados pela Equação 5.

$$
\{X\}_{k+1}=\{X\}_{k}+\alpha\{d\}_{k}
$$

na qual o vetor $\mathrm{d}$, de mesma ordem do vetor $X$, indica a direção de busca, também conhecida como direção de descida nos problemas de minimização, e o escalar positivo $\alpha$ indica o comprimento do passo a ser dado na direção determinada, de modo a minimizar a função nesta direção. Como se observa, um problema com n variáveis, uma vez determinada a direção de busca, passa a ser tratado como um problema unidimensional, função exclusivamente do escalar $\alpha$. O ponto obtido definirá um novo vetor $X$, aqui representado pelo sub-índice $k+1$, sendo $k$ o número da iteração anterior. Todo o procedimento descrito é então repetido a partir do novo ponto, até que um critério de parada pré-definido seja atendido.

Os métodos de otimização variam basicamente pela forma de determinação, a cada iteração, da direção de descida e do tamanho do passo a ser dado nesta direção. Neste trabalho, optou-se pela utilização de um método heurístico, conhecido como método do politopo ou busca simplex (NELDER e MEAD, 1965), especialmente indicado devido ao alto grau de complexidade que acarretaria a determinação das derivadas das funções. Além disso, por meio de experimentos anteriores, verificou-se que o método é adequado ao número de variáveis envolvidas na otimização geométrica de pontes na forma proposta no presente trabalho.

O método trabalha com a construção de um simplex, um polígono com $n+1$ vértices, sendo $n$ o número de variáveis de projeto. Calculado o valor da função objetivo em cada um dos vértices, o de pior valor é refletido sobre o centróide dos demais vértices. $\mathrm{O}$ valor da função nesse novo ponto é determinado, podendo ainda ser efetuados os movimentos de expansão ou contração. Com esse novo vértice substituindo o de pior valor, procede-se de forma iterativa, reduzindo o tamanho do simplex de acordo com critérios pré-definidos e prossegundo até que o critério de convergência seja atendido. Em resumo, trata-se de um procedimento relativamente simples, de fácil implementação computacional e com número reduzido de parâmetros de controle quando comparado a heurísticas 
mais recentes como o método dos algorítmos genéticos. Apesar de antigo, o método ainda é amplamente empregado nas mais diversas aplicações. A título de ilustração, um estudo de Wright (2012) mostrava que, de janeiro a maio de 2012, mais de dois mil artigos publicados naquele ano já haviam sido disponibilizados no Google Acadêmico utilizando o método desenvolvido por Nelder e Mead. Além disso, diversar variações vêm sendo propostas com o objetivo de ampliar a aplicação do método a problemas com grande número de variáveis. Dentre estes, destaca-se o estudo de Gao e Chan (2010), no qual são empregado valores variáveis para os parâmetros de contração, reflexão e expansão.

Pela difícil ocorrência, em casos práticos de engenharia, de problemas de otimização não sujeitos a qualquer tipo de restrição, estas são consideradas pela técnica de penalização. Segundo esta técnica, as soluções não factíveis, ou seja, que não atendem às condições impostas no problema, são incrementadas de um valor o qual, usualmente, consiste na multiplicação da restrição violada por um fator de penalização. A desvantagem deste procedimento consiste no fato de que, caso o processo iterativo seja interrompido precocemente, o resultado obtido não será necessariamente uma solução factível para o problema formulado. Em contrapartida, dispensa-se a obrigatoriedade do conhecimento a priori de um ponto inicial factível, ponto este que pode ser de difícil determinação.

2.2 Formulação do Método dos Deslocamentos para a obtenção dos esforços

Para efeito de análise computacional de estruturas, os métodos matriciais demonstram-se como ferramentas de grande eficiência. Dentre os principais métodos para a análise de estruturas reticuladas, o Método dos Deslocamentos é o de emprego mais difundido, basicamente pela fácil sistematização do processo para a determinação dos deslocamentos e esforços (SORIANO, 1983).

O Método dos Deslocamentos envolve primeiramente a resolução de um sistema de equações da forma:

$$
[\mathrm{K}]\{\mathrm{U}\}=\{\mathrm{F}\}
$$

Em que: K, F e U representam, respectivamente: a matriz de rigidez global da estrutura (função das características do material, da seção transversal e do comprimento de cada elemento, bem como de sua orientação no plano ou espaço relativamente aos demais elementos); o vetor de forças nodais (ou forças nodais equivalentes), determinadas pelo carregamento aplicado e pelo comprimento do elemento carregado; e o vetor de deslocamentos nodais, este último contendo as incógnitas do sistema. Uma vez determinados os deslocamentos, calculam-se os esforços nos extremos de cada elemento a partir da rigidez do próprio elemento $\left(\mathrm{r}_{\mathrm{i}}\right)$, dos deslocamentos dos nós extremos do elemento $(\delta)$ e das ações eventualmente aplicadas ao longo do mesmo $\left(\mathrm{P}_{0}\right)$, ou ações de engastamento perfeito:

$$
\begin{gathered}
\{\mathrm{P}\}_{\mathrm{i}}=[\mathrm{r}]_{\mathrm{i}}\{\delta\}_{\mathrm{i}}+\left\{\mathrm{P}_{0}\right\}_{\mathrm{i}} \\
\text { 3- FORMULAÇÃO DO PROBLEMA DE } \\
\text { OTIMIZAÇÃO }
\end{gathered}
$$

Com o objetivo de determinar a relação ótima entre os vãos de vigas de pontes, buscando tanto a redução como a uniformização dos esforços, consideraram-se as posições dos apoios internos como variáveis do problema, de forma a, em se supondo a existência de uma certa liberdade na locação destes apoios, evitar a sub-utilização da capacidade resistente da estrutura. A substituição de um carregamento estático, usualmente empregado nos problemas de otimização, por uma carga móvel, acarreta em um grau de complexidade bastante superior, uma vez que, correspondendo às infinitas posições que podem ser assumidas pela carga, existem infinitos valores para os esforços em cada seção da estrutura (PFEIL, 1979).

A análise de estruturas submetidas a cargas móveis pode ser decomposta em duas etapas distintas, quais sejam: a determinação das linhas de influência e a superposição do carregamento. A linha de influência de um determinado efeito consiste em um diagrama auxiliar que pode, entre outros, ser definido como por Souza e Antunes (1994), segundo os quais a linha de influência (s) de um determinado efeito $E_{C}$, de uma seção $C$ de uma estrutura, para carga percorrendo uma determinada linha $\mathrm{S}$ associada a essa estrutura, é o valor $E_{\mathrm{C}}(\mathrm{s})$ correspondendo a uma carga adimensional unitária, vertical e orientada de cima para baixo, aplicada na posição definida pela coordenada s sobre S. Uma vez obtida a linha de influência, e considerando válido o princípio da superposição dos efeitos, a obtenção dos efeitos extremos provocados por um carregamento qualquer pode ser facilmente efetuada. Supondo-se um carregamento usual composto por cargas concentradas e ou distribuídas, a determinação do máximo efeito pode ser efetuada, segundo SÜSSEKIND (1987), de acordo com a Equação 8:

$$
\mathrm{E}_{\mathrm{C}}=\sum \mathrm{P}_{\mathrm{i}} \eta_{\mathrm{i}}+\mathrm{q} \Omega
$$

Em que: $\mathrm{P}_{\mathrm{i}}$ representa o valor da i-ésima carga concentrada, $\eta_{i}$ a ordenada correspondente à carga $P_{i}, q 0$ valor do carregamento uniformemente distribuído e $\Omega$ a área, no diagrama de linhas de influência, correspondente à extensão do carregamento. Observa-se que os efeitos máximos são obtidos sem a necessidade de se recorrer às equações de equilíbrio de forças ou de compatibilidade de deformações.

Devido aos graus de dificuldade relativos apresentados por cada uma das duas etapas envolvidas na determinação dos efeitos em pontes, o presente estudo limitou-se à obtenção das linhas de influência de esforços, uma vez que o passo seguinte constitui-se unicamente em um procedimento de aplicação direta da expressão anterior, não necessitando portanto da experiência do engenheiro calculista ou de conhecimento prévio adicional. 
Para a elaboração da formulação apresentada para a otimização de vigas contínuas de pontes tendo como variáveis de projeto as posições dos apoios, algumas considerações iniciais foram efetuadas de forma a simplificar parcialmente a análise sem o comprometimento da aplicabilidade prática do procedimento proposto. Supondo-se a adoção de uma única dimensão para a seção transversal ao longo de toda a estrutura, optou-se por não incluir na análise a otimização de seção transversal dos elementos. O objetivo, portanto, foi o de propiciar de forma direta apenas uma redução nos esforços extremos, tendo-se porém a possibilidade de, a partir dos resultados da análise, efetuar o redimensionamento da seção transversal. Cabe enfatizar que, desta forma, uma eventual economia deve-se unicamente à redistribuição dos esforços na estrutura, e portanto sem comprometimento da segurança. Adicionalmente, devido às características apresentadas pelo carregamento aplicado para a determinação das linhas de influência de esforços, estas assumirão obrigatoriamente uma configuração final simétrica (exceto na presença de restrições laterais). Desta forma, se pode partir de soluções iniciais igualmente simétricas de modo a acelerar a convergência.

As variáveis do problema foram tomadas como as coordenadas dos apoios em relação ao primeiro nó da estrutura, situado à esquerda dos demais. Pelo fato do comprimento total da viga contínua permanecer inalterado, bem como pela imposição de apoios fixos nos extremos (momentos fletores nulos nestes pontos), o vetor das variáveis de projeto exclui estes nós extremos, sendo portanto de dimensão total NNO-2, onde NNO corresponde ao número total de nós do modelo, sendo o número de elementos igual a NNO-1. Assim, o comprimento $L$ de um determinado elemento $i$ da viga pode ser determinado conforme Equação 9:

$$
L_{i}=x_{i+1}-x_{i}
$$

\section{Conforme ilustrado na Figura 1.}

Figura 1 - Otimização em função da posição dos apoios: estrutura genérica

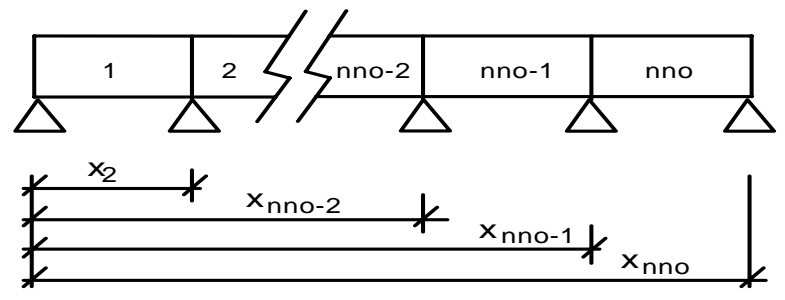

A formulação idealizada partiu da consideração de que, como a altura da seção transversal de uma viga é definida em função do maior momento fletor, a busca da redução e uniformização nos valores destes esforços facultaria tanto a obtenção de menores dimensões para a seção como o melhor aproveitamento da armadura disposta ao longo dos elementos. Como estes objetivos são perseguidos pela alteração nas posições relativas dos apoios, estas posições definem o conjunto das variáveis de projeto. De forma a definir o posicionamento final ou ótimo foi objetivada a minimização do quadrado das máximas ordenadas não positivas, em cada tramo, das linhas de influência de esforços de flexão relativas a cada um dos apoios internos da estrutura. Como restrição é imposta a igualdade das ordenadas dos vãos imediatamente adjacentes a cada apoio interno, no diagrama de linhas de influência correspondente a este apoio. Caracterizado o problema, este é formulado como:

Min $\sum\left(\eta_{i, k}^{2}\right) i=1$, NELM, $k=2$, NNO-1

Sujeito a

$$
\begin{aligned}
& \eta_{\mathrm{i}, \mathrm{i}}-\eta_{\mathrm{i}-1, \mathrm{i}}=0 \\
& \mathrm{x}_{\mathrm{k}}{ }^{\mathrm{l}} \leq \mathrm{x}_{\mathrm{k}} \leq \mathrm{x}_{\mathrm{k}}{ }^{\mathrm{u}}
\end{aligned}
$$

Em que NELM é o número total de elementos ou vãos e NNO o número total de nós ou apoios da estrutura.

Ao se formular o problema de otimização em função da posição de alguns nós na estrutura, os esforços solicitantes nos elementos passam a ser influenciados de forma determinante por qualquer variação na posição destes nós, pela alteração no comprimento dos elementos. Desta forma, tanto as matrizes de rigidez como os vetores que contêm as ações de engastamento perfeito, ao envolverem explicitamente as distâncias relativas entre seus nós extremos, permitiriam a obtenção direta das derivadas dos coeficientes em relação a cada um dos nós. Já para a obtenção das derivadas dos deslocamentos necessita-se de um processo um pouco mais trabalhoso do ponto de vista computacional, por serem estes deslocamentos funções implícitas das posições dos nós. Desta forma, optou-se pela implementação de um método de busca direta para a obtenção da direção de descida, de modo a simplificar o código do programa. Por se tratar de estruturas com relativamente poucos apoios, e portanto de um número reduzido de variáveis do ponto de vista de otimização, o tempo de processamento resultou igualmente pouco significativo, não sendo portanto dada maior atenção a este aspecto no presente trabalho.

\section{4 - APLICAÇÕES NUMÉRICAS}

São apresentados, a seguir, os resultados obtidos a partir da análise de vigas com número de apoios crescente. Estas análises buscaram tanto verificar a validade da formulação proposta como a identificação de relações que possam subsidiar a determinação das relações ótimas entre os vãos.

\subsection{Viga contínua com dois vãos}

Desta forma, ilustra-se o problema descrito inicialmente pela análise de uma viga constituída por um único apoio interno. Sabe-se que, em função das características do carregamento, a geometria final desta estrutura deve ser obrigatoriamente simétrica, com o eixo de simetria coincidente com o apoio central. No entanto, de forma a destacar a sensibilidade dos esforços a pequenas variações na posição ótima, este apoio central foi deslocado em relação a esta posição. Na Figura 2 são ilustrados diagramas de Linhas de Influência (LI) dos valores obtidos para o momento fletor no apoio interno, tanto para o apoio 
no eixo de simetria (vãos de mesmo comprimento) como para um deslocamento de 10 por cento na posição do apoio central em relação a este eixo, da esquerda para a direita.

Figura 2 - Exemplo 1: LI dos momentos fletores no apoio central, a) no eixo de simetria e b) deslocado.
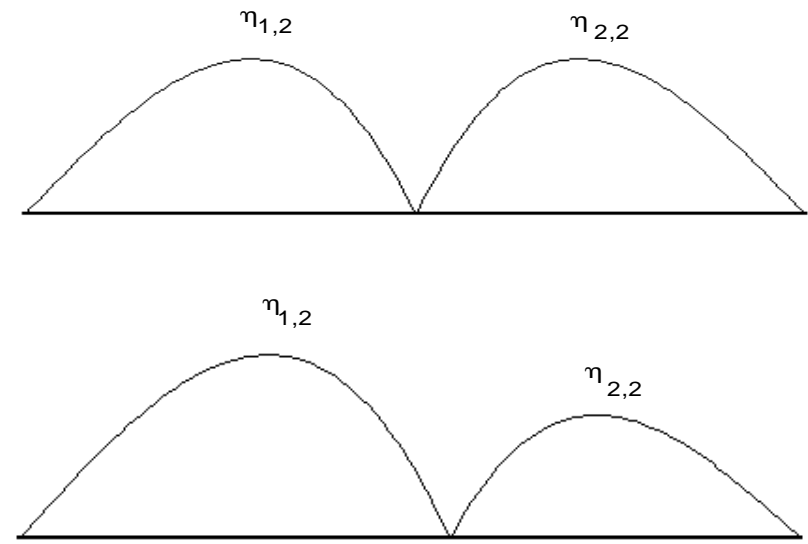

Observa-se na Figura 2 uma grande variação, na ordenada da linha de influência, do valor do máximo esforço de flexão (denominado $\eta_{i, 2}$ segundo a notação apresentada). Ainda mais significativa é a variação relativa entre os esforços máximos para a carga unitária situada à esquerda e à direita do apoio central $\left(\eta_{1,2}\right.$ e $\eta_{2,2}$, respectivamente), no caso do apoio deslocado.

Os resultados decorrentes da movimentação do apoio central para uma viga de comprimento total igual a 20 metros e inércia constante encontram-se listados na Tabela 1 , com $\mathrm{x}_{2}$ representando a posição do segundo apoio (apoio interno) de acordo com a convenção já empregada na Figura 1. Os casos indicados pelas letras B a D representam, respectivamente, $\mathrm{x}_{2}$ variando dez, vinte e trinta por cento sua posição em relação à configuração simétrica (caso A). Os asteriscos indicam uma inversão no sentido dos esforços.

Tabela 1 - Exemplo 1: Variação dos momentos fletores máximos no apoio interno

\begin{tabular}{ccccccc}
\hline Caso & $\mathrm{x}_{2}(\mathrm{~m})$ & $\mathrm{x}_{2} / \mathrm{L}$ & $\begin{array}{c}\eta_{1,2} \\
(\mathrm{~m})\end{array}$ & $\begin{array}{c}\eta_{2,2} \\
(\mathrm{~m})\end{array}$ & $\begin{array}{c}\eta_{1,2} / \\
\eta_{2,2}\end{array}$ & $\begin{array}{c}\eta_{1,2} / \\
\eta_{1,2 \mathrm{~A}}\end{array}$ \\
\hline $\mathrm{A}$ & 10,00 & 0,50 & 0,9600 & 0,9600 & 1,000 & 1,000 \\
\hline $\mathrm{B}$ & 11,00 & 0,55 & 1,1591 & 0,7777 & 1,490 & 1,207 \\
\hline $\mathrm{C}$ & 12,00 & 0,60 & 1,3854 & 0,6094 & 2,273 & 1,443 \\
\hline $\mathrm{D}$ & 13,00 & 0,65 & 1,3846 & $-0,6148$ & $*$ & $*$ \\
\hline
\end{tabular}

Como pode ser observado na Tabela 1, para um pequeno deslocamento relativo na posição do apoio central afastando-se do ótimo (caso A), tem-se uma grande variação no valor correspondente ao máximo esforço. De acordo com a última coluna, verifica-se que a máxima ordenada na linha de influência sofre um aumento superior ao dobro da variação no deslocamento, sendo que a partir de um determinado ponto a diferença entre as rigidezes relativas entre os vãos torna-se tão grande que ocorre uma inversão no sentido do esforço de flexão. Adicionalmente, observa-se que a variação no valor dos máximos efeitos entre os dois vãos é ainda mais significativa. Exemplificando-se com dados da tabela, para uma alteração em 10 por cento na posição do apoio central a partir do ponto de ótimo tem-se uma diferença relativa de 49 por cento nos valores das coordenadas dos dois tramos e de $20 \%$ no valor da coordenada no tramo 1 (última coluna). Como o dimensionamento da seção transversal da estrutura normalmente é efetuado predominantemente em função do maior valor do efeito ao longo da viga, apresentam-se indicações de uma grande economia na medida em que a configuração da estrutura aproxima-se da ótima, independentemente do material utilizado e sem detrimento da segurança.

Os valores correspondentes aos máximos efeitos (tração na face inferior, designada por momento fletor positivo), bem como sua sensibilidade, também foram analisados, conforme ilustrado na Tabela 2. Observa-se que a máxima ordenada não sofre uma variação tão significativa quanto à da linha de influência obtida para os momentos fletores no apoio central.

Tabela 2 - Exemplo 1: Variação dos momentos fletores no centro dos vãos

\begin{tabular}{ccccccc}
\hline Caso & $\mathrm{x}_{2}(\mathrm{~m})$ & $\mathrm{x}_{2} / \mathrm{L}$ & $\begin{array}{c}\eta_{1,2} \\
(\mathrm{~m})\end{array}$ & $\begin{array}{c}\eta_{2,2} \\
(\mathrm{~m})\end{array}$ & $\begin{array}{c}\eta_{1,2} / \\
\eta_{2,2}\end{array}$ & $\begin{array}{c}\eta_{1,2} / \\
\eta_{1,2 \mathrm{~A}}\end{array}$ \\
\hline $\mathrm{A}$ & 10,00 & 0,50 & 2,0640 & 2,0640 & 1,000 & 1,000 \\
\hline $\mathrm{B}$ & 11,00 & 0,55 & 2,2334 & 1,8870 & 1,183 & 1,082 \\
\hline $\mathrm{C}$ & 12,00 & 0,60 & 2,3961 & 1,7049 & 1,405 & 1,160 \\
\hline $\mathrm{D}$ & 13,00 & 0,65 & 2,5521 & 1,5203 & 1,678 & 1,236 \\
\hline
\end{tabular}

Cabe destacar que as diferenças apresentadas se referem apenas à máxima ordenada em cada vão. Uma vez que os carregamentos usuais são compostos também por cargas distribuídas, como indicado na equação (8), as diferenças nos efeitos finais são um pouco distintas dos valores indicados na tabela, uma vez que as áreas não variam em relação direta com as correspondentes ordenadas.

\subsection{Viga contínua com três vãos}

O segundo exemplo apresentado consiste em uma viga contínua com três vãos, ou quatro apoios, e inércia constante. O comprimento total da estrutura é de 30 metros, podendo, para efeito de análise cada vão ser subdividido de maneira uniforme em 10 trechos, conforme a prática corrente.

Inicialmente são apresentados na Tabela 3 os esforços de flexão, relativos à carga unitária, obtidos para os apoios internos da estrutura. A exemplo da viga anteriormente analisada, apenas os valores das máximas ordenadas são objeto de comparação. Na tabela, unicamente a posição do primeiro apoio interno é indicada, bem como as ordenadas dos vãos adjacentes a este apoio, devido à simetria da estrutura. O caso A representa a disposição de apoios gerando três vãos de mesmo comprimento, a qual é tomada como estrutura de referência para efeito de análise dos resultados, uma vez que consiste em uma configuração de adoção bastante freqüente. Já os casos B e C representam os valores próximos do ótimo considerada a precisão de 
centímetros. No caso D a precisão é levada à casa dos milímetros.

Tabela 3 - Exemplo 2: Variação dos efeitos em função da posição dos apoios

\begin{tabular}{|c|c|c|c|c|c|c|c|}
\hline Caso & $\begin{array}{c}\mathrm{X}_{2} \\
(\mathrm{~m})\end{array}$ & $x_{2} / L$ & $\begin{array}{l}\mathrm{x}_{2} / \\
\mathrm{x}_{2} *\end{array}$ & $\begin{array}{l}\eta_{1,2} \\
(\mathrm{~m})\end{array}$ & $\begin{array}{c}\eta_{2,2} \\
\text { (m) }\end{array}$ & $\begin{array}{c}\eta_{1,2} / \\
\eta_{2,2}\end{array}$ & $\begin{array}{l}\eta_{\max } / \\
\eta_{\max }\end{array}$ \\
\hline A & 10,00 & 0,3333 & 1,046 & 1,024 & 0,800 & 1,280 & 1,000 \\
\hline B & 9,560 & 0,3186 & 0,999 & 0,915 & 0,916 & 0,988 & 0,895 \\
\hline C & 9,570 & 0,3190 & 1,001 & 0,917 & 0,913 & 1,004 & 0,896 \\
\hline D & 9,562 & 0,3187 & 1,000 & 0,915 & 0,915 & 1,000 & 0,894 \\
\hline
\end{tabular}

Da Tabela 3 pode-se verificar a variação tanto no valor da posição relativa do apoio como nas ordenadas correspondentes na linha de influência, na medida em que se parte da configuração usual até chegar ao ponto de ótimo. Observa-se, com base na penúltima coluna, que as máximas ordenadas de esforços de flexão imediatamente adjacentes ao apoio considerado deslocável ao longo do eixo da estrutura, passam a ser idênticas no caso D (atendimento às restrições de igualdade), ao passo que na configuração inicial assumiam uma diferença de 28 por cento. Desta forma, a economia relativa entre estas duas situações é de aproximadamente 10,6 por cento (última coluna). Destaca-se que a diferença percentual indicada refere-se a um deslocamento na posição do apoio em apenas 438 milímetros no sentido dos extremos da estrutura, o que representa menos de 1,5 por cento do comprimento total da viga. Cabe observar que, em termos práticos, uma variação desta ordem na posição do apoio dificilmente seria impedida, quer por questões de ordem física ou mesmo estética.

De forma a facilitar a observação dos efeitos resultantes de deslocamentos relativos de maior grau, são apresentados na Tabela 4 os valores correspondentes à variações de até dois metros, ou 6,67 por cento, na posição dos apoios internos, em ambos os sentidos.

Tabela 4 - Exemplo 2: Variação dos efeitos em função da posição dos apoios

\begin{tabular}{ccccccc}
\hline Caso & $\mathrm{x}_{2}(\mathrm{~m})$ & $\mathrm{x}_{2} / \mathrm{x}_{2}{ }^{*}$ & $\begin{array}{c}\eta_{1,2} \\
(\mathrm{~m})\end{array}$ & $\begin{array}{c}\eta_{2,2} \\
(\mathrm{~m})\end{array}$ & $\begin{array}{c}\eta_{1,2} / \\
\eta_{2,2}\end{array}$ & $\begin{array}{c}\eta_{\max } / \\
\eta_{\max \mathrm{A}}\end{array}$ \\
\hline $\mathrm{E}$ & 8,00 & 0,836 & 0,6164 & 1,3781 & 0,447 & 1,346 \\
\hline $\mathrm{F}$ & 9,00 & 0,941 & 0,8066 & 1,0694 & 0,754 & 1,044 \\
\hline $\mathrm{A}$ & 10,00 & 1,046 & 1,0240 & 0,8000 & 1,280 & 1,000 \\
\hline $\mathrm{H}$ & 11,00 & 1,150 & 1,2767 & 0,5516 & 2,314 & 1,247 \\
\hline $\mathrm{H}$ & 12,00 & 1,255 & 1,5833 & 0,3214 & 4,9262 & 1,546 \\
\hline
\end{tabular}

\subsection{Viga contínua com quatro vãos}

Para este exemplo apresentam-se os resultados obtidos para a máxima ordenada das linhas de influência de momento fletor nos apoios para uma viga com três apoios internos e comprimento total igual a 40 metros. Para esta situação, igualmente às demais, compara-se (Tabela 5) os valores obtidos na configuração ótima, designada por caso $\mathrm{B}$, com uma configuração inicial que considera os apoios como dispostos de forma eqüidistante. Observou-se que esta disposição, ao tornar o apoio 3 coincidente com o eixo de simetria da estrutura, acelera enormemente o processo de convergência, uma vez que a posição final deste apoio é também coincidente com o eixo de simetria. Desta forma, de acordo com a formulação do problema, tem-se que $\eta_{2,3}$ é igual a $\eta_{3,3}$, ou seja, uma das restrições é sempre atendida, razão pela qual apenas o primeiro destes valores é apresentado na Tabela 5.

Tabela 5 - Exemplo 3: Variação dos efeitos em função da posição dos apoios internos

\begin{tabular}{ccccccc}
\hline Caso & $\mathrm{x}_{2}(\mathrm{~m})$ & $\mathrm{x}_{3}(\mathrm{~m})$ & $\begin{array}{c}\eta_{1,2} \\
(\mathrm{~m})\end{array}$ & $\begin{array}{c}\eta_{2,2} \\
(\mathrm{~m})\end{array}$ & $\begin{array}{c}\eta_{2,3} \\
(\mathrm{~m})\end{array}$ & $\begin{array}{c}\eta_{\max } / \\
\eta_{\max , \mathrm{A}}\end{array}$ \\
\hline $\mathrm{A}$ & 10,00 & 20,00 & 1,0285 & 0,7885 & 0,8571 & 1,0000 \\
\hline $\mathrm{B}$ & 9,33 & 20,00 & 0,9019 & 0,9026 & 0,9064 & 0,8825 \\
\hline
\end{tabular}

Para a estrutura analisada verifica-se que a máxima ordenada no diagrama de linhas de influência ocorre não mais no segundo apoio para a carga no primeiro vão da estrutura, mas sim no terceiro apoio para a carga no segundo ou terceiro vãos, sendo estas máximas ordenadas as comparadas na última coluna da tabela, independentemente do ponto onde ocorrem.

\subsection{Efeitos verificados com a consideração de cargas acidentais}

Nos exemplos anteriores os resultados representam somente os efeitos referentes às linhas de influência. De forma a verificar os efeitos gerados pela consideração de carregamentos de maior complexidade, apresentam-se a seguir os resultados obtidos na análise de vigas, com a consideração do trem-tipo classe $45(450 \mathrm{kN})$, definido pela Norma Técnica Brasileira ABNT NBR 7188:1984. Para fins de comparação adotaram-se vigas com apoios eqüidistantes em 10m, com número de apoios variando entre 3 e 9. Com o mesmo objetivo foram consideradas diferentes larguras para a ponte. Na Tabela 6 são apresentadas as relações entre os máximos momentos negativos obtidos para a estrutura ótima e os máximos momentos negativos obtidos para a viga com seus apoios eqüidistantes.

Tabela 6 - Efeitos verificados com a consideração de cargas acidentais para pontes com número de apoios e larguras variáveis

\begin{tabular}{ccccc} 
& \multicolumn{4}{c}{ Largura da ponte (m) } \\
\cline { 2 - 5 } & 7,00 & 8,00 & 9,00 & 10,00 \\
\hline NNO & \multicolumn{4}{c}{ Mmax(-) ótimo / Mmax(-) inicial } \\
\hline 3 & 1,0000 & 1,0000 & 1,0000 & 1,0000 \\
\hline 4 & 0,9644 & 0,9668 & 0,9706 & 0,9712 \\
\hline 5 & 0,9330 & 0,9354 & 0,9372 & 0,9389 \\
\hline 6 & 0,9210 & 0,9227 & 0,9243 & 0,9259 \\
\hline 7 & 0,9099 & 0,9114 & 0,9129 & 0,9143 \\
\hline 8 & 0,9026 & 0,9040 & 0,9054 & 0,9066 \\
\hline 9 & 0,8800 & 0,8806 & 0,8812 & 0,8817 \\
\hline
\end{tabular}

Verifica-se, por meio dos resultados listados na Tabela 6, que os máximos momentos negativos para as estruturas ótimas são inferiores aos obtidos nas estruturas originais, independentemente do número de apoios e da largura da ponte. Percebe-se também que, à medida em que o número de apoios aumenta, maior o grau de economia em relação aos apoios dispostos de forma equidistante. 


\subsection{Considerações sobre o processo de otimização}

São apresentados, a seguir, alguns dados adicionais sobre a implementação computacional e a forma de obtenção dos resultados computacionais. Em todas as análise efetuadas, foram considerados os fatores de reflexão, expansão e retração usualmente empregados no método, quais sejam: 1 , 2 e 1/2, respectivamente. O simplex inicial foi gerado com aresta de dimensão unitária, com fator de penalização $r$ igual a10. Este fator multiplica as restrições de igualdade, e seu resultado é somado à função objetivo original.

Na Tabela 7 são apresentados os resultados das iterações iniciais, bem como a solução otimizada, relativos ao exemplo 3 (viga com quatro vãos, ou três apoios internos). Na tabela, iter indica o número da iteração, $F(x)$ é o valor da função objetivo e Fpen o valor da função penalizada.

Tabela 7 - Processo iterativo do método de otimização (exemplo 3)

\begin{tabular}{ccccccc}
\hline iter & vert & $\mathrm{x}_{2}(\mathrm{~m})$ & $\mathrm{x}_{3}(\mathrm{~m})$ & $\mathrm{x}_{4}(\mathrm{~m})$ & $\mathrm{F}(\mathrm{x})$ & $\mathrm{Fpen}$ \\
\hline 1 & 1 & 10,000 & 20,000 & 30,000 & 2,882 & 7,682 \\
\hline $\mathbf{1}$ & $\mathbf{2}$ & $\mathbf{1 0 , 9 4 3}$ & $\mathbf{2 0 , 2 3 6}$ & $\mathbf{3 0 , 2 3 6}$ & $\mathbf{3 , 0 5 1}$ & $\mathbf{1 1 , 3 4 1}$ \\
\hline 1 & 3 & 10,236 & 20,943 & 30,236 & 3,064 & 10,134 \\
\hline 1 & 4 & 10,236 & 20,236 & 30,943 & 2,828 & 7,088 \\
\hline 2 & 1 & 10,000 & 20,000 & 30,000 & 2,882 & 7,682 \\
\hline 2 & 2 & 9,371 & 20,550 & 30,550 & 2,770 & 6,400 \\
\hline $\mathbf{2}$ & $\mathbf{3}$ & $\mathbf{1 0 , 2 3 6}$ & $\mathbf{2 0 , 9 4 3}$ & $\mathbf{3 0 , 2 3 6}$ & $\mathbf{3 , 0 6 4}$ & $\mathbf{1 0 , 1 3 4}$ \\
\hline 2 & 4 & 10,236 & 20,236 & 30,943 & 2,828 & 7,088 \\
\hline$\ldots$ & $\ldots$ & $\ldots$ & $\ldots$ & $\ldots$ & $\ldots$ & $\ldots$ \\
\hline 42 & \multicolumn{7}{c}{9,330} & 20,000 & 29,670 & 2,447 & 2,467 \\
\hline \multicolumn{7}{c}{}
\end{tabular}

Uma vez que o problema possui três variáveis, é gerado um politopo com quatro vértices, sendo o primeiro deles a solução inicial fornecida (todos os vãos de mesmo comprimento) e os demais obtidos de modo a constituir o simplex com vértices equidistantes. A pior solução (em negrito) foi refletida gerando uma nova solução, que passou a constituir a melhor solução vigente. A expansão não melhorou esta solução. A nova pior solução (vértice 3 da iteração 2, em negrito) foi também refletida, e assim sucessivamente, até o atendimento ao critério de parada (tamanho do simplex). Cabe observar que o valor da função penalizada (última linha da Tabela 7) difere um pouco do valor da função não penalizada, indicando que os valores das máximas ordenadas das linhas de influência em cada vão não são idênticos (como pode-se verificar na Tabela 5, caso B). Um critério de penalização mais restritivo poderia reduzir a diferença observada, no entanto o número de iterações aumentaria de forma significativa. Além disso, o número total de iterações se mostrou altamente dependente do tamanho do simplex inicial. Um simplex grande necessita de um número grande de iterações até ser reduzido. Por outro lado, um simplex muito pequeno também não é vantajoso caso a solução inicial se encontre afastada do ponto de ótimo.

\section{5 - CONCLUSÕES E CONSIDERAÇÕES FINAIS}

Este estudo, de forma global, teve como objetivo verificar a aplicabilidade da otimização dos esforços em estruturas de pontes analisadas pelo modelo de viga quando se permite a variação relativa nas posições dos apoios. Neste sentido, apesar das possíveis limitações de ordem física, bem como das simplificações inerentes à própria formulação proposta para o tratamento do problema, observa-se que os resultados obtidos fornecem indícios de uma economia significativa no consumo de materiais estruturais.

A utilização de técnicas de otimização associada à análise de estruturas tem sido alvo de um número muito grande de estudos nas últimas décadas. Contudo, a carência de exemplos práticos apresentados na literatura técnica, aliada à restrita divulgação das potencialidades da otimização estrutural, com problemas fundamentalmente voltados à redução do peso da estrutura, pouco tem encorajado maiores investimentos na aplicação da otimização a problemas usualmente encontrados.

Com o intuito de preencher uma lacuna em relação ao cálculo de estruturas correntes foi estudada a viabilidade do desenvolvimento de uma ferramenta auxiliar ao projetista. Acredita-se que o estudo efetuado neste sentido pode ser considerado promissor, possibilitando ainda a associação de outros objetivos que não apenas os abrangidos no presente trabalho, tais como a otimização simultânea da posição dos apoios e da altura da seção.

Ainda que não constem de forma explícita da formulação desenvolvida verificou-se que, a exemplo da otimização geométrica aplicada a estruturas submetidas a carregamento fixo, uma série de outros efeitos favoráveis são obtidos. Dentre estes efeitos, destaca-se a uniformização e redução também nos valores dos esforços cisalhantes devido à uniformização dos momentos fletores sobre os apoios.

Pretende-se que os estudos desenvolvidos se constituam não unicamente em uma demonstração de um campo alternativo para a aplicação de técnicas de otimização à análise estrutural, mas sim em um passo efetivo no sentido do desenvolvimento de ferramentas computacionais para a busca tanto de uma melhor solução em problemas usuais como também para a resolução de problemas de maior complexidade.

\section{REFERÊNCIAS}

Associação Brasileira de Normas Técnicas - ABNT. NBR 7188: carga móvel em ponte rodoviária e passarela de pedestre, Rio de Janeiro, 1984.

COHN, M. Z.; DINOVITZER, A. S. Application of structural optimization. Journal of Structural Engineering, ASCE, v. 120, n. 2, p. 617-50, Feb, 1994. GAO, F.; HAN, L. Implementing the Nelder-Mead simplex algorithm with adaptive parameters. Comput. Optim. Appl. v. 51, n. 1, p. 259-277, Jan. 2012.

IMAM, M. H.; AL-SHIRI, M. Optimum topology of structural supports. Computers \& Structures, v. 61, n. 1, p. 147-54, 1996. 
KIRSCH, U. Feasibility and optimality in structural design. Computers \& Structures, v. 41, n. 6, p. 134956, 1991.

KRIPKA, M.; ANTUNES, H. M. C. C. Optimum support positions in R-C beams. In: STEVEN, G.P. et al. Structural Optimisation (Proceedings of the Australasian Conference on Structural Optimisation, Sidney, Australia, 11-13 Feb 1998) Victoria, Oxbridge Press, p. 367-374, 1998.

KRIPKA, M.; ANTUNES, H. M. C. C. Optimum Support Positions in Building Grillages. In: Hernandez, S.; Brebbia, C.A.. (Org.). Computer Aided Optimum Design of Structures VII. 1 ed. Southampton: WIT Press, v. 01, p. 117-126, 2001.

NELDER, J. A.; MEAD, R. A simplex Method for Function Minimization. The Computer Journal, v. 7, n. 4, p. 308-313, Jan., 1965.

PFEIL, W. Pontes em concreto armado. Rio de Janeiro, LTC., 1979.

SORIANO, H. L. Análise matricial de estruturas reticuladas em computadores de pequeno porte. Rio de Janeiro, UFRJ, 1983.
SOUZA, J. C. A. O.; ANTUNES, H. M. C. C. Cargas móveis em estruturas lineares. São Carlos, EESC/USP, 1997.

SPILLERS, W. R. Iterative design for optimal geometry. Journal of Structural Division, Proceedings of the American Society of Civil Engineers, ASCE, v. 101, n. ST7, p. 1435-1442, July, 1975.

SÜSSEKIND, J. C. Curso de Análise Estrutural. Rio de Janeiro, Ed. Globo, 1987.

WANG, D. Optimal design of structural support positions for minimizing maximal bending moment. Finite Elements in Analysis and Design, v. 43, n. 2, p. 95-102, 2006.

WANG, D.; JIANG, J. S.; ZHANG, W. H. Optimization of support positions to maximize the fundamental frequency of structures. Int. J. Numer. Meth. Engng, v. 61, p. 15841602, 2004.

WRIGHT, M. H. Nelder, Mead, and the other simplex method. Documenta Mathematica, special volume (Optimization Stories), p. 271-276, 2012. 